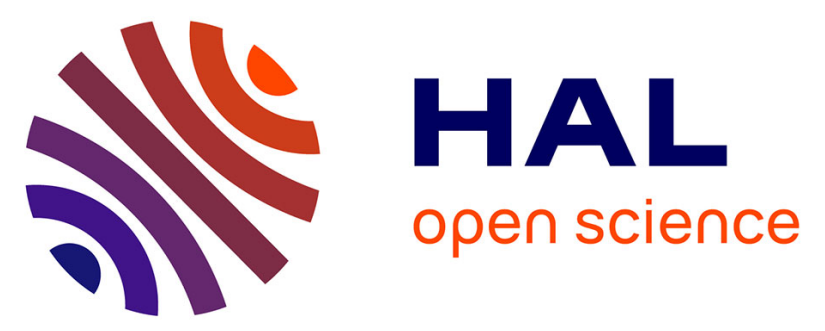

\title{
Cardiovascular correlates of emotional state, cognitive workload and time-on-task effect during a realistic flight simulation
}

\author{
Antonio R. Hidalgo-Muñoz, Damien Mouratille, Nadine Matton, Mickael \\ Causse, Yves Rouillard, Radouane El-Yagoubi
}

\section{To cite this version:}

Antonio R. Hidalgo-Muñoz, Damien Mouratille, Nadine Matton, Mickael Causse, Yves Rouillard, et al.. Cardiovascular correlates of emotional state, cognitive workload and time-on-task effect during a realistic flight simulation. International Journal of Psychophysiology, 2018, 128, pp.62-69. 10.1016/j.ijpsycho.2018.04.002 . hal-01761909

\section{HAL Id: hal-01761909 \\ https://hal-enac.archives-ouvertes.fr/hal-01761909}

Submitted on 30 Jul 2018

HAL is a multi-disciplinary open access archive for the deposit and dissemination of scientific research documents, whether they are published or not. The documents may come from teaching and research institutions in France or abroad, or from public or private research centers.
L'archive ouverte pluridisciplinaire HAL, est destinée au dépôt et à la diffusion de documents scientifiques de niveau recherche, publiés ou non, émanant des établissements d'enseignement et de recherche français ou étrangers, des laboratoires publics ou privés. 


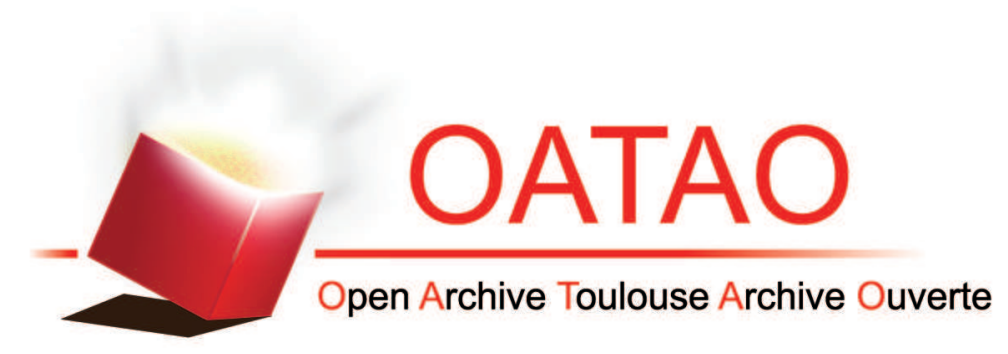

\section{Open Archive Toulouse Archive Ouverte (OATAO)}

OATAO is an open access repository that collects the work of some Toulouse researchers and makes it freely available over the web where possible.

This is an author's version published in: https://oatao.univ-toulouse.fr/20487

Official URL : http://doi.org/10.1016/j.jpsycho.2018.04.002

\section{To cite this version :}

Hidalgo-Muñoz, Antonio R. and Mouratille, Damien and Matton, Nadine and Causse, Mickaël and Rouillard, Yves and El-Yagoubi, Radouane Cardiovascular correlates of emotional state, cognitive workload and time-on-task effect during a realistic flight simulation. (2018) International Journal of Psychophysiology, 128. 62-69. ISSN 0167-8760

Any correspondence concerning this service should be sent to the repository administrator: tech-oatao@listes-diff.inp-toulouse.fr 


\title{
Cardiovascular correlates of emotional state, cognitive workload and time- on-task effect during a realistic flight simulation
}

\author{
Antonio R. Hidalgo-Muñoz ${ }^{\mathrm{a}, *}$, Damien Mouratille ${ }^{\mathrm{a}}$, Nadine Matton ${ }^{\mathrm{b}}$, Mickaël Causse ${ }^{\mathrm{c}}$, \\ Yves Rouillard $^{\mathrm{b}}$, Radouane El-Yagoubi ${ }^{\mathrm{a}}$

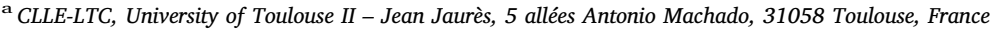 \\ b École National de l'Aviation Civile, 7 Édouard-Belin, 31055 Toulouse, France \\ ${ }^{\mathrm{c}}$ Institut Supérieur de l'Aéronautique et de l'Espace, 10 Édouard-Belin, 31055 Toulouse, France
}

\begin{tabular}{ll} 
& A B S T R A C T \\
\cline { 2 - 2 } $\begin{array}{l}\text { Keywords: } \\
\text { Cognitive workload } \\
\text { Emotion }\end{array}$ & $\begin{array}{l}\text { In aviation, emotion and cognitive workload can considerably increase the probability of human error. An } \\
\text { Flight simulation }\end{array}$ \\
Heart rate & heart rate variability (HRV) of 21 private pilots were analysed during two realistic flight simulator scenarios. \\
Heart rate variability & Emotion was manipulated by a social stressor and cognitive workload with the difficulty of a secondary task. Our \\
Neuro-ergonomics & results confirmed the sensitivity of the HR to cognitive demand and training effects, with increased HR when the \\
& task was more difficult and decreased HR with training (time-on-task). Training was also associated with an \\
& increased HRV, with increased values along the flight scenario time course. Finally, the social stressor seemed to \\
provoke an emotional reaction that enhanced motivation and performance on the secondary task. However, this & was not reflected by the cardiovascular activity.
\end{tabular}

\section{Introduction}

Investigating the emotional state and the cognitive workload experienced by operators becomes a primordial concern in numerous domains where the human factor is critical. This is the case in aviation, where pilots are commonly exposed to different sources of emotional and cognitive stressors (Blogut, 2015). Hence, not surprisingly, approximately $70 \%$ of accidents are due, at least partially, to erroneous decisions or distraction of the crew (Salas et al., 2010). Therefore, integrating an efficient and objective online monitoring to assess the emotional and/or the cognitive variations into the cockpit would be largely desirable. For this purpose, reliable and cost-effective physiological measures are required.

According to Scherer (2005), an emotion can be defined as "an episode of interrelated, synchronized changes in the states of all or most of the [...] organismic subsystems in response to the evaluation of an external or internal stimulus event as relevant to major concerns of the organism". On the other hand, the concept of cognitive workload deals with the link between the demand of the tasks and the available resources (Wickens, 2008). As is well known, emotion and cognition both have an impact on the autonomic nervous system (e.g. Causse et al., 2010; Critchley, 2005; Critchley et al., 2013). However, the analysis of their combined effects while handling complex tasks, e.g. the control of a plane, has not been considered in great detail in literature and remains challenging. The research mainly or even exclusively focuses on one of these factors by neglecting a possible interaction between them. The manipulation of the cognitive workload often leads to variations in the emotional state, and disentangling their respective impact remains complex. For example, increased task difficulty is also considered to elicit psychological stress (Wang et al., 2005). Similarly, an intensive cognitive activity may yield a high level of workload without eliciting a high level of stress, and a high level of stress might occur when the workload is low. In addition, even if emotion and cognition are supposed to be underpinned by distinct structural brain networks (e.g. limbic system vs. prefrontal cortex, Gaillard and Wientjes, 1994), the probability of observing analogous modulations on peripheral physiological activity is high (Mandrick et al., 2016).

In the particular case of emotion, the effect of anxiety, i.e. an unpleasant feeling of fear over anticipated events, during simulation training has been explored by Tichon et al. (2014). This study found larger muscle activation for anxious states, while ocular measurements did not show significant variations, except for the fixation times, which were shorter in anxiety scenarios. Differently, in Causse et al. (2013), the influence of emotion on pilot decision-making has been studied by using functional magnetic resonance imaging, necessarily out of a cockpit. In this case, the emotion was generated by rewarding the

\footnotetext{
* Corresponding author.

E-mail address: arhidalgom@gmail.com (A.R. Hidalgo-Muñoz).
} 
participants according to the relevance of their landing decisions in different situations of uncertainty. Participants who exhibited riskier decisions showed lower activity in the right dorsolateral prefrontal cortex, suggesting a disruptive effect of biased financial incentive on the rational decision-making neural network. In a similar aeronautical context, Causse et al. (2011) showed that increased emotional arousal due to the financial incentive generates an increased heart rate (HR). Interestingly, Allsop and Gray (2014) analysed the effect of anxiety generated by social stress on attention and gaze behaviour in aviation. The increment in the level of arousal caused impairment in attentional control, as reflected by the saccade rate and increased muscle activation. Similarly, Gray et al. (2016) applied the same method to elicit stress on the participants in order to disentangle the particular influences on specific attentional processes.

On the other hand, the effects of cognitive (otherwise referred to as "mental") workload and its objective measurements is a topic raising increased interest in diverse domains, such as ergonomics in general (Young et al. (2015) for a review) or aviation in particular (Vecchiato et al., 2016). For instance, regarding the measures of the central nervous system, electroencephalography (EEG) has been widely used to assess cognitive overload and to discriminate specific psychological processes involved in multi-task resolution with different purposes (Puma et al., 2018; Stikic et al., 2014). Another application, but using peripheral measures, is found in Gaetan et al. (2015). The authors have studied the physiological markers referring to distinct levels of workload in helicopter pilots by using electromyography and skin conductance, although definitive conclusions could not be drawn due to large individual differences. Moreover, the usefulness of the respiratory signals has been studied to verify the correlation with subjective measures. Muth et al. (2012) have shown that the respiratory sinus arrhythmia (RSA) index can predict scores on the NASA-Task Load Index questionnaire. The authors tested RSA as a real-time measure of cognitive workload in participants completing a performance-based selection test developed by the U.S. Navy.

Recently, emerging studies started analysing mental workload under stressing situations in tasks requiring a high level of performance. Specifically, in the field of aeronautics, Mandrick et al. (2016), by means of pupillometry, functional near infrared spectroscopy (fNIRS) and cardiovascular measurements, investigated the physiological correlations of human performance under high level of workload when threatened by unpredictable auditory stressors. Their results showed that higher task difficulty (higher $n$-back level) degraded the performance and induced an increased tonic pupil diameter, HR and activity in the lateral prefrontal cortex, as well as decreased phasic pupil response and heart rate variability (HRV). The condition of emotional stress did not affect the performance, but at the expense of a psychophysiological cost, as demonstrated by a lower phasic pupil response, and a greater HR and prefrontal activity.

Despite the numerous possibilities of physiological measures linked to emotion and cognition, electrocardiography (ECG) is still considered among the most suitable options, even for complex computational models integrating emotional and cognitive factors, for example in Besson et al. (2012), providing powerful and discriminant features for classifier implementation. As mentioned in several of the studies above, HR and HRV are two fundamental ECG parameters (Massaro and Pecchia, 2016) often taken into account in studies focusing on the relationship between cardiovascular activity and emotion and/or cognition in ergonomics (Durantin et al., 2014). Wei et al. (2014) showed the suitability of HRV measures to distinguish among three levels of mental workload, as illustrated by a reduction of the standard deviation of RR intervals when the task was more difficult, consistent with results from the study of Causse et al. (2011). Mansikka et al. (2016) studied HR and HRV features for differentiating between sub-standard performance and high-performance approaches in fighter pilots. The results showed a decrease in HRV-based values in high performance approaches. Following this line, other applications can be found in car drivers, where
HR and HRV have received a particular attention (Heine et al., 2017; Mehler et al., 2012; Stuiver et al., 2014).

Given the non-stationary nature of HR and HRV measures, the timeon-task factor is one important aspect to take into account. For instance, Luque-Casado et al. (2016) demonstrated a decrement in HRV values with time-on-task, which would be linked to vigilance decrement or mental fatigue under high cognitive demand. This concern has also been raised in other studies dealing with either training (e.g., Socha et al., 2015 suggest that pilots with more extensive training sessions exhibit lower values on the short-term power spectrum of ECG) or emotional habituation (Feda and Roemmich, 2016; Sevenster et al., 2015).

The results above are promising and encourage a deeper exploration of the ECG markers to inspect, in flight, the relation between emotional state and cognitive demand. In this study, we examine how the presence of a social stressor and the difficulty of a secondary task modulate the cardiovascular activity of pilots. According to literature (Causse et al., 2011; Wei et al., 2014; Mandrick et al., 2016), an increase in HR alongside a decrease in HRV is expected when arousal and/or cognitive workload increases. Moreover, to check whether training and/or habituation has a relevant effect on the physiological measures (LuqueCasado et al., 2016), different intra-condition segments are considered.

\section{Materials and methods}

\subsection{Participants}

Twenty-one healthy volunteers were recruited from the French Civil Aviation University (ENAC) in Toulouse, France. All participants had normal auditory acuity and normal or corrected to normal vision. None of them declared a history of severe medical treatment, neither psychological disorder nor any cardiac or neurological trouble except one participant who suffered from extra-systoles and had to be discarded. The 20 remaining participants were included in the physiological analysis (only male; $22.7 \pm 3.7$ years). All participants gave written informed consent two days before carrying out the experiment in accordance with the local ethical board committee. The study complied with the Declaration of Helsinki for human experimentation. All of them were in possession of the Private Pilot License, had at least a flying experience of 50 flight hours (141.3 $\pm 139.5 \mathrm{~h})$, were familiar to simulator environments and moreover were fluent in English.

\subsection{Experimental setting}

In order to reach an acceptable external validity, our experiment has been designed to simulate as closely as possible a real flight situation. The experiment took place in an AL-50 simulator (Fig. 1), located in a room with artificial dimmed lighting and kept at a comfortable temperature. The experiment consisted in completing two dual-task scenarios. A scheme of the experimental protocol is shown in Fig. 2. Each dual-task scenario required the simultaneous accomplishment of a preestablished flight plan with the flight simulator and a secondary task based on target stimulus discrimination, aiming at modulating the level of cognitive workload (low or high). Furthermore, the dual-task scenarios were carried out in two different conditions (with or without social stressor). This $2 \times 2$ design enabled to separately assess the effects of both emotional and cognitive manipulations.

\subsubsection{Flight scenarios}

Two flight scenarios analogous in terms of difficulty, one for each emotional state condition of the experiment (with or without social stressor), were designed by an expert instructor. Each flight lasted approximately $35 \mathrm{~min}$ from take-off to landing and there was a break of 20 min between them. The established flight paths required various changes in heading, speed and altitude specifications to vary the course. More specifically, the second flight scenario included the same 


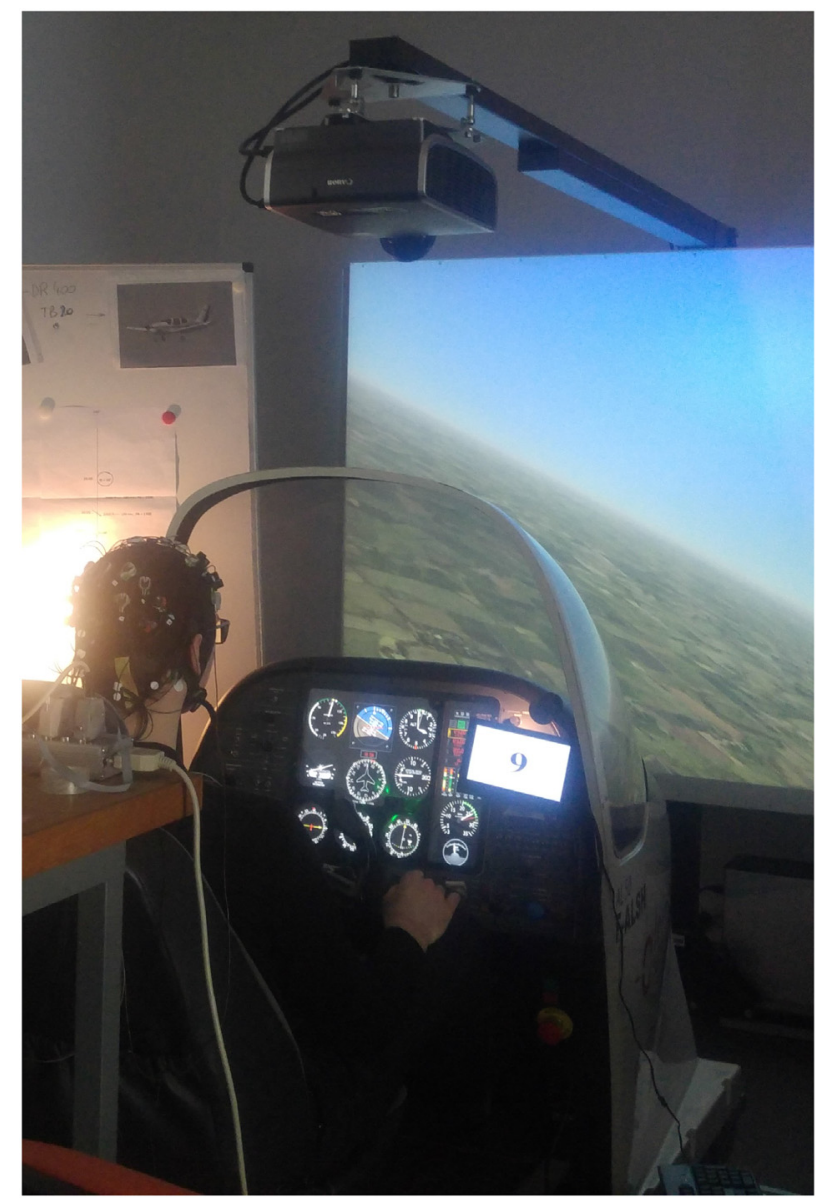

Fig. 1. Participant in the AL-50 simulator.

instructions as the first one, but performed in a different order and in the opposite direction. For instance, whereas in the first scenario there was a $360^{\circ}$ turn to the right at $5 \mathrm{~min}$ from take-off, in the second scenario, there was a $360^{\circ}$ turn to the left after $30 \mathrm{~min}$. The angle values to change the heading were counterbalanced and randomly placed within each scenario and the equivalent difficulty of the two scenarios was verified by two expert instructors. There were two altitude changes, one speed reduction and the same number of heading specifications (degrees of heading) in both scenarios. A strict timing for the flight instructions was specified in the flight plan. During all flight scenarios, the information about the various manoeuvers to be performed was made available to the participant. Favourable weather conditions were set and unexpected technical events were not included.

The trajectories and parameters from both flight tasks were recorded and examined by a professional experimenter in order to verify the completion of the instructions. Several flight parameters such as speed (measured in knots), heading (degrees) and altitude $(\mathrm{m})$ were collected continuously during the simulations. The performance was considered as acceptable when the deviations of the expected parameters fell into a margin, as used by the flight's assessors at ENAC. Any deviation greater than \pm 5 units from the requested flight parameter, was flagged as an error (i.e. excessive deviation). A conservative threshold of five units permitted to reveal subtle differences in performance over time, independently of pilot expertise. A sampling rate of $1 \mathrm{~Hz}$ was applied to assess the number of errors. A global performance was also computed by adding the partial performances of all parameters.

\subsubsection{Emotional state manipulation}

We chose to modulate the emotional state using the method depicted in the study of Allsop and Gray (2014). During the first dual-task scenario (including a complete flight plan and the two versions of the cognitive secondary task explained below), considered as the Low Arousal (LA) condition, the participant was left alone in the flight simulator. On the contrary, during the execution of the second dual-task scenario, considered as the High Arousal (HA) condition, the participant was filmed by two cameras and his voice was recorded with a microphone. Moreover, the participant was thoroughly monitored by two researchers and was supposedly involved in a competition with the other participants. This mock competition would consist in a public nominative classification of the individual results from the experiment. The truth about the fact that the competition and filming was unreal was disclosed at the end of the experiment.

Note that, for the HA condition, the arousal could be increased due to the motivation and/or to the social stress of feeling evaluated. Two questionnaires (French version) were used to find out the origin of the emotional state for ulterior analysis out of the scope of this study. The former, the Competitive State Anxiety Inventory-2R (Martinent et al., 2010) to measure the anxiety linked to the competition, was delivered just before the HA condition, whereas the latter, the State-Trait Anxiety Inventory (form Y) (Spielberger et al., 1983) to measure the general anxiety, was given when the second dual-task scenario was finished. In any case, distinguishing the concepts of emotion and stress was not a goal of the present work, but rather it was employing the social stress as an emotional factor, as suggested in the literature (Blanchard et al., 1998; Lazarus, 2006; Staal, 2004).
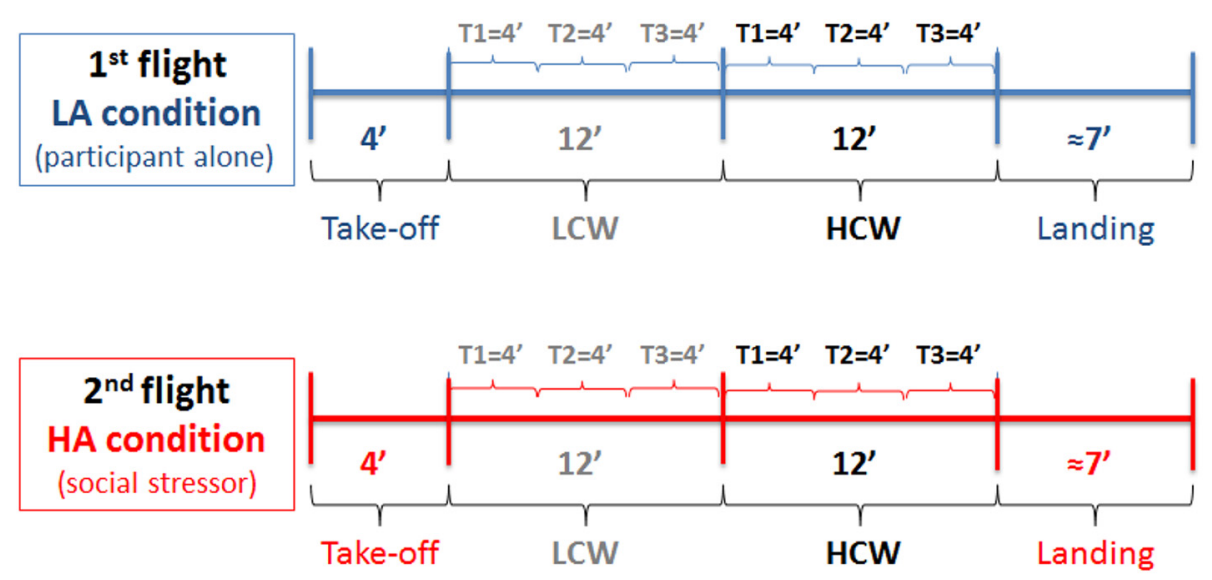

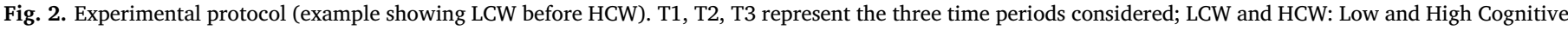
Workload, respectively; LA and HA: Low and High Arousal, respectively. 


\subsubsection{Cognitive workload manipulation}

A 7-inch touchscreen computer (TFT LCD, resolution $800 \times 480$ ) with built-in speakers was employed to implement the secondary task. The touchscreen was integrated on the right side of the cockpit panel and was easily accessible by the pilots (see Fig. 1). Essentially, this task consisted of touching the screen as quickly as possible after hearing some numbers integrated among Air Traffic Control (ATC) audio instructions (in English). These ATC instructions were not related to the flight plan and were used only as distractors, making the environment more realistic.

This secondary task was implemented using E-prime 2.0 (CPsychology Software Tool, Inc., PA, USA) and was presented during the cruise only, excluding take-off and landing periods. It was subdivided in two phases of the same duration (twelve minutes) separated by a bell sound, permitting to manipulate the additional cognitive workload, as follows.

- Low Cognitive Workload (LCW): The participant was instructed to press the screen if the perceived numbers met a simple rule. For the first dual-task scenario, the number ought to be greater than five, while for the second dual-task scenario, the rule consisted of pressing the screen if the perceived number was even. Simultaneously to the heard numbers, a series of random numbers written in black were displayed on the screen. The two types of numbers (heard and visualized) could present equal or different values. In the LCW condition, these displayed numbers ought to be ignored.

- High Cognitive Workload (HCW): Similarly to the other phase, the participant was instructed to press the screen according to the value of the perceived numbers. However, for this condition, the attribute value on which the attention had to be oriented depended on the colour of the visually perceived numbers (displayed on the screen). In other words, the colour of the visualized number indicated if the attribute to evaluate was the magnitude (pressing if it was greater than five) or the parity (pressing if the number was even) of the heard number. The colours used were red and green for the first dual-task scenario, and magenta and blue for the second dual-task scenario. For this HCW condition, the participant was instructed to look at the screen after each heard number, in order to check the colour, and thus the attribute to evaluate. For example, if a number displayed during the first scenario was written in red and, simultaneously, a heard number was superior to 5 , the participant was instructed to touch the screen. This instruction requires mental flexibility and increased working memory/attentional demands.

There was no time lapse between LCW and HCW conditions, except an auditory stimulus (bell) indicating the switch. The presentation order of LCW and HCW conditions were counterbalanced among the participants to avoid possible different allocations of cognitive resources due to the overload imbalance. Actually, potential order effects were considered in preliminary analyses and no significant results were obtained. Participants showing a performance that is considered too low, i.e. $<50 \%$ of correct responses, implying a misunderstanding, were discarded for behavioural-based mean comparisons.

\subsection{ECG measurement}

ECG signal was recorded (sampling rate $=1 \mathrm{MHz}$ ) throughout the entire experiment by placing two electrodes on the clavicle and left pectoral of the participants. BrainVision Recorder 1.21 (CBrain Products $\mathrm{GmbH}$, Gilching, Germany) was used for signal acquisition. The ground reference signal was taken from the EEG-fNIRS montage, employed for ulterior analysis. All ECG features were computed in Matlab 2016a (CThe Mathworks, Inc., MA, USA) by taking three 4minute segments referring to the different parts of the experiment as detailed in previous sections (LCW and HCW for LA and HA).
The signal pre-processing included a band-pass filtering with 0.5 and $40 \mathrm{~Hz}$ cut-off frequencies by a 4th-order zero-phase Butterworth filter to reject high frequency noise and baseline wander artefacts. A visual inspection was carried out to check signal quality. The meaning of every feature was as follows:

- HR: Heart rate corresponds to the mean number of beats (by counting the R-peaks) per minute (bpm) within the 4-min segment.

To evaluate HRV:

- SD (RR): Standard deviation of R-R interval lengths for each 4-min segment.

- RMSSD: Root mean squared of the successive differences between adjacent R-R intervals. Usually, it is associated with fast (parasympathetic) variability.

- pNN- $x$ : Proportion of R-R intervals which differ more than $x$ ms with respect to the adjacent previous $\mathrm{R}-\mathrm{R}$ interval. When $x=\mathrm{SD}$, the $\mathrm{SD}$ refers to the standard deviation of R-R interval lengths taking the whole recording as reference, i.e. it deals with an intra-subject specific measure.

\subsection{Statistical analysis}

All statistical analyses were carried out using STASTICA version 12 (CStatSoft Inc., OK, USA). Normality was first checked for the parameter value distributions by means of the Shapiro-Wilk's. Then, an analysis of variance (ANOVA) of repeated measures was performed including three within-subject factors: emotion (2 levels of arousal defining the emotional state: LA and HA) $\times$ cognition $(2$ levels of cognitive workload: LCW and HCW) $\times$ time-on-task intra-condition (3 levels: $\{1:=(0-4 \mathrm{~min}), 2:=(4-8 \mathrm{~min}), 3:=(8-12 \mathrm{~min})\})$. To check for any statistically significant difference between conditions, a maximum $p$-value of .05 was set. Greenhouse-Geisser's correction was applied if sphericity could not be assumed. Post hoc analysis was based on HSD Tukey criterion. In short, a $2 \times 2 \times 3$ analysis was carried out. According to the results reported in Causse et al. (2017) concerning the workload effect, the required sample size was $N=20$ and the expected standardized effect with power $=0.80$ would be 0.675 .

\section{Results}

\subsection{Flight performance}

Concerning the performance in the primary task, i.e. the flight plan, two participants were discarded due to issues with data recording.

Neither main effects of cognition or emotion, nor interaction between both factors were observed on flight performance, regardless of the criteria used: overall performance (emotion: $F(1,18)=1.56$, $p=.23$; cognition: $\quad F(1,18)=0.26, \quad p=.61 ; \quad$ interaction: $F$ $(1,18)=1.92, p=.18$ ), speed criteria (emotion: $F(1,18)=0.94$, $p=.34 ; \quad$ cognition: $\quad F(1,18)=0.75, \quad p=.40 ; \quad$ interaction: $F$ $(1,18)=1.39, p=.25$ ), altitude criteria (emotion: $F(1,18)=1.28$, $p=.27 ; \quad$ cognition: $\quad F(1,18)=1.99, \quad p=.84 ; \quad$ interaction: $F$ $(1,18)=0.12, p=.73$ ) and heading criteria (emotion: $F(1,18)=1.02$, $p=.33 ; \quad$ cognition: $\quad F(1,18)=0.82, \quad p=.38 ; \quad$ interaction: $F$ $(1,18)=4.16, p=.06)$.

\subsection{Performance in the secondary task}

The performance in the secondary task was measured for eleven participants. The other participants were discarded due to technical problems in data acquisition (the connection between touchscreen and PC was not operating as desired for seven participants) or inefficient performances (three participants reached $<50 \%$ of accuracy in at least one of the four conditions, i.e. emotion $\times$ cognition). 


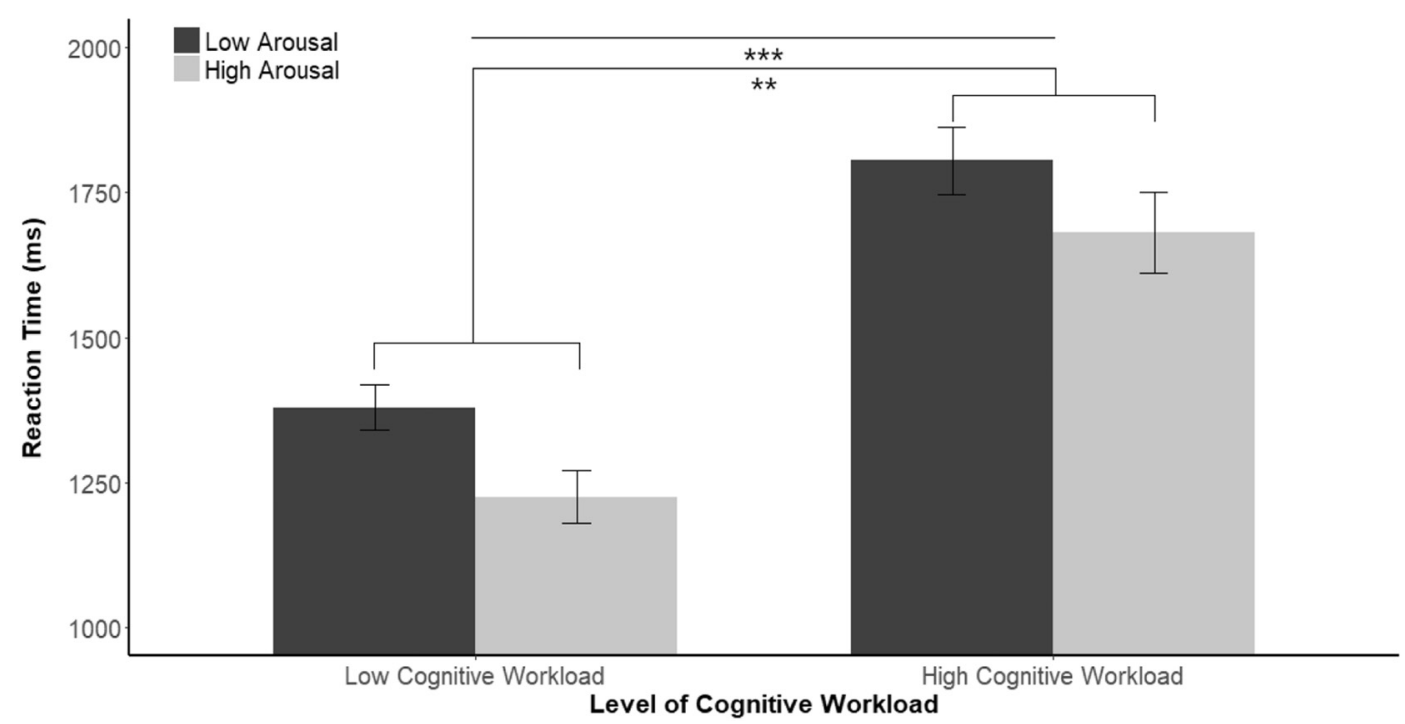

Fig. 3. Reaction times for the secondary task for Low and High Cognitive Workload for Low and High Arousal conditions. ${ }^{* *} p \leq .01 ;{ }^{* * *} p \leq .001$.

The results showed significant main effects for both emotion and cognition factors, with faster reactions times (RT) (less time to respond) for HA than for LA condition: $F(1,10)=13.64, p=.004, \eta_{p}{ }^{2}=0.58$, and for LCW compared to HCW condition: $F(1,10)=66.02, p<.001$, $\eta_{p}{ }^{2}=0.87$. No interaction between cognition and emotion was found $(F$ $(1,10)=0.33, p=.58)$. Fig. 3 represents RT means.

Regarding the accuracy, two sources of error have been considered: commission errors when unexpected responses were given, and omission errors when the subject did not react to the target stimulus. A global accuracy of $94.6 \%$ was obtained on average. No significant effect of emotion and cognition was found on global errors (i.e. the addition of commission and omission errors) (for the emotion factor: $F$ $(1,10)=4.96, p=.051, \eta_{p}{ }^{2}=0.33 ;$ for the cognition factor: $F$ $(1,10)=0.88, p=.37$; for the interaction term: $F(1,10)=2.13$, $p=.18)$. However, a main effect of emotion on omission errors was found, i.e. between LA and HA condition, $F(1,10)=5.15, p=.05$, $\eta_{p}{ }^{2}=0.34$; showing a higher number of omissions in LA compared to HA ( $10.8 \%$ and $5.68 \%$, respectively), yielding to a reduction of $52.6 \%$ of the omission rate under HA (see Fig. 4).

\subsection{ECG features}

\subsubsection{Emotion and cognition factors}

A main effect of cognition was found for HR: $F(1,19)=4.56$, $p=.046, \quad \eta_{p}{ }^{2}=0.19, \quad$ showing a greater value for HCW $(M=86.55 \mathrm{bpm}, S D=15.18)$ compared to LCW $(M=85.14 \mathrm{bpm}$, $S D=15.47)$ condition $(p=.013)$. No main effect of emotion $(F$ $(1,19)=1.05, p=.31)$ and no interaction was found $(F(1,19)=1.72$, $p=.21$ ). No significant results were found for the HRV features.

Table 1 shows the statistics (mean \pm standard deviations) for all ECG features for both emotional state and cognitive workload conditions, averaging the results of the three 4-min segments intra-condition.

\subsubsection{Time-on-task factor}

Concerning the time-on-task factor while secondary task was carried out, a main effect was found for HR: $F(2,38)=11.69, p<.001$, $\eta_{p}{ }^{2}=0.38$, Intraclass correlation (ICC) $=0.994$; RMSSD: $F$ $(2,38)=5.59, \quad p=.01, \quad \eta_{p}{ }^{2}=0.23, \quad$ ICC $=0.933 ; \quad$ pNN-20: $\quad F$ $(2,38)=10.67, \quad p<.001, \quad \eta_{p}^{2}=0.35, \quad$ ICC $=0.987 ; \quad$ pNN-50: $\quad F$ $(2,38)=6.80, p=.002, \quad \eta_{p}{ }^{2}=0.26, \quad$ ICC $=0.988$ and pNN-SD: $F$ $(2,38)=6.78, p=.003, \eta_{p}{ }^{2}=0.26$, ICC $=0.976$. Post hoc analyses

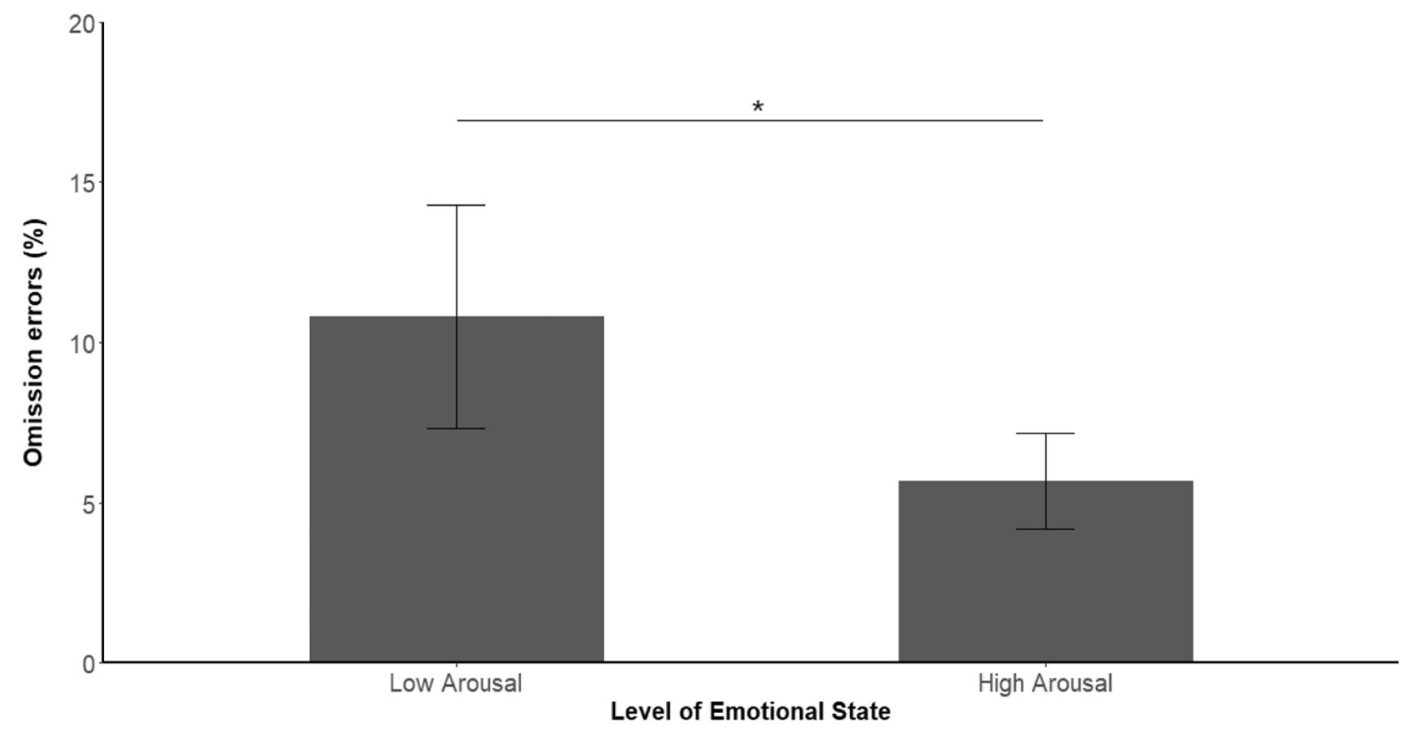

Fig. 4. Omissions errors for the secondary task for Low and High Arousal conditions. ${ }^{*} p \leq .05$. 
Table 1

Mean and standard deviation values of ECG features and correlation values.

\begin{tabular}{|c|c|c|c|c|}
\hline \multirow[t]{2}{*}{ Feature } & \multicolumn{2}{|l|}{ Low Arousal } & \multicolumn{2}{|l|}{ High Arousal } \\
\hline & LCW & HCW & LCW & HCW \\
\hline HR (bpm) & $84.87 \pm 15.47$ & $85.67 \pm 15.10$ & $85.42 \pm 15.60$ & $87.42 \pm 15.33$ \\
\hline SD(RR) (s) & $0.050 \pm 0.025$ & $0.052 \pm 0.024$ & $0.050 \pm 0.015$ & $0.049 \pm 0.017$ \\
\hline RMSSD & $0.033 \pm 0.002$ & $0.029 \pm 0.013$ & $0.031 \pm 0.016$ & $0.03 \pm 0.0015$ \\
\hline pNN-20 & $0.42 \pm 0.019$ & $0.42 \pm 0.18$ & $0.41 \pm 0.20$ & $0.40 \pm 0.20$ \\
\hline pNN-50 & $0.12 \pm 0.13$ & $0.11 \pm 0.12$ & $0.12 \pm 0.14$ & $0.12 \pm 0.16$ \\
\hline pNN-SD & $0.103 \pm 0.078$ & $0.098 \pm 0.068$ & $0.1 \pm 0.079$ & $0.10 \pm 0.095$ \\
\hline
\end{tabular}

revealed that the HR slowed down significantly from the first segment $(M=89.19, \quad S D=8.93)$ compared to the middle $(M=86.06$, $S D=6.44)$ and the last one $(M=81.87, S D=2.32)(p=.025$; $p<.001$, respectively). An opposed tendency, i.e. increasing values over time, was found for RMSSD, which increased significantly from the first segment $(M=0.034, S D=0.011)$ compared to the middle $(M=0.037, S D=0.007)$ and the last one $(M=0.044, S D=0.005)$ ( $p=.026 ; p=.012$, respectively). In the case of $\mathrm{pNN}-x$ features, a similar result to RMSSD was obtained. For instance, for pNN-20, an increase between the first $(M=0.46, S D=0.17)$ and the last segment $(M=0.60, S D=0.03)$ was observed $(p<.001)$.

An interaction between the time-on-task and cognition was found in HR: $F(2,38)=3.39, p=.04, \eta_{p}{ }^{2}=0.15$, pNN-20: $F(2,38)=6.11$, $p=.01, \eta_{p}{ }^{2}=0.24$, pNN-50: $F(2,38)=5.66, p=.01, \eta_{p}{ }^{2}=0.23$ and pNN-SD: $F(2,38)=7.71, p=.001, \eta_{p}{ }^{2}=0.29$. For HR, the post hoc analysis showed significant differences between the beginning of the HCW condition and the two other segments, yielding almost $4 \mathrm{bpm}$ of total reduction in this condition. Analogous results, but in the opposed direction, were found for the pNN- $x$ values with a $22 \%$ increase in $\mathrm{pNN}$ 20 value during HCW conditions. Neither an interaction between emotion and time-on-task, nor a triple interaction, among the three factors, were statistically significant for any feature. For visualization purposes, the most relevant results for HR and pNN-20 are depicted in Figs. 5 and 6, respectively.

\section{Discussion}

Before discussing the results, it is fair to mention the comment of Mansikka et al. (2016) reminding that while a simulator mission can be designed to be mentally extremely demanding, it will inherently lack the stressors of an actual flying mission such as the sense of risk and the fear of collision or injury of death. According to this conclusive argument, the level of stress induced by a flight simulator has to be considerate as moderate (Jorna, 1993).

\subsection{Effects of emotion and cognition on the performance in the flight simulation and the secondary task}

Behavioural results supported that both emotional and cognitive factors significantly affected behaviour. On the one hand, the social stressor seemed to provoke an emotional reaction that enhanced motivation and performance in the secondary task as revealed by the decrease in omission errors and the difference in RT between LA and HA. On the other hand, the increased cognitive workload of this secondary task seems to be deleterious to performance by inducing an increase in RT.

On the contrary, flight performances (primary task) were equivalent within every condition. Thus, variations on RT were not associated with a prioritization of the secondary task to the detriment of the flight performance. In other words, participants prioritized this latter task even when the secondary task was more complex or when social stress was present.

\subsection{Effects of emotion and cognition on ECG}

Although the behavioural results suggest that we successfully increased the emotional arousal, this fact was not evidenced by the ECG measures. Probably, the lack of main effect of emotion on HR was partly due to an insufficient emotional activation, which could have been stronger with a direct sensorial stimulation. For example, using aversive auditory stimuli, Mandrick et al. (2016) demonstrated effects of emotion on HR. Nevertheless, in agreement with this research work and others (e.g., Heine et al., 2017), HR was impacted by the cognitive demand of the secondary task, being faster when difficulty increased

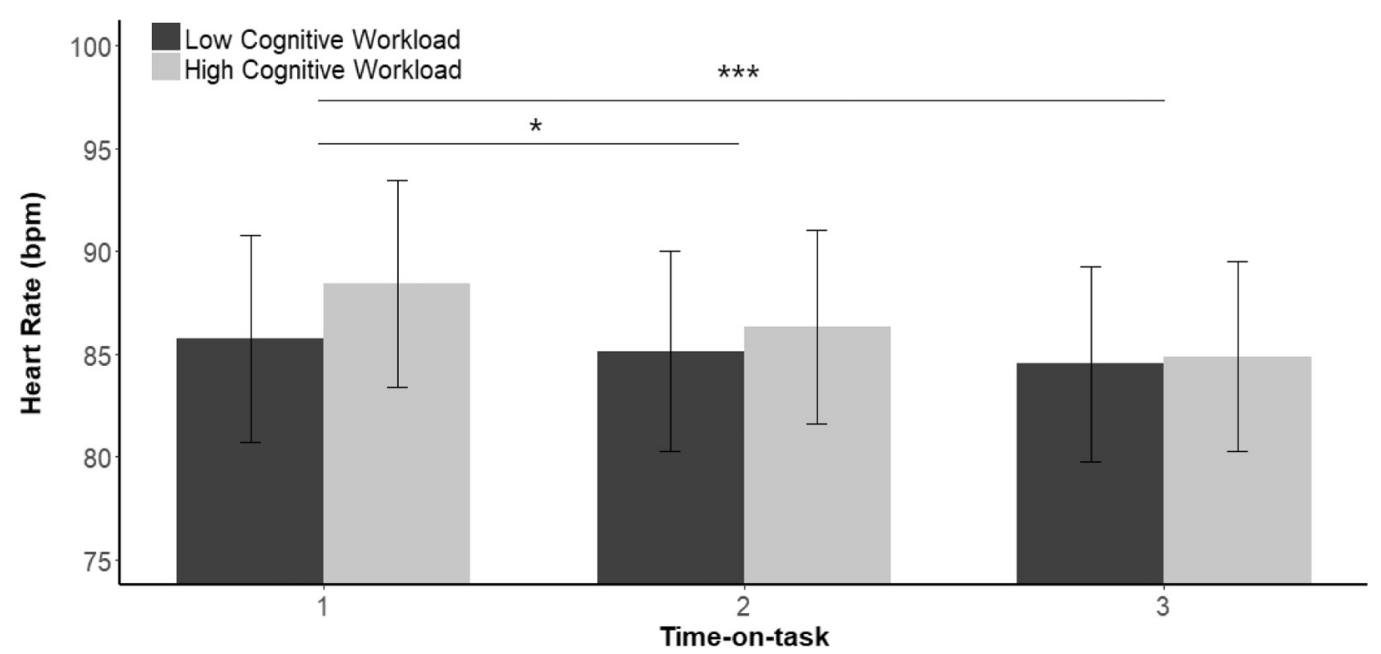

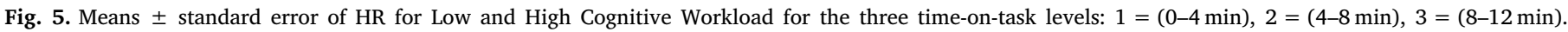
${ }^{*} p \leq .05 ; * * * p \leq .001$. 


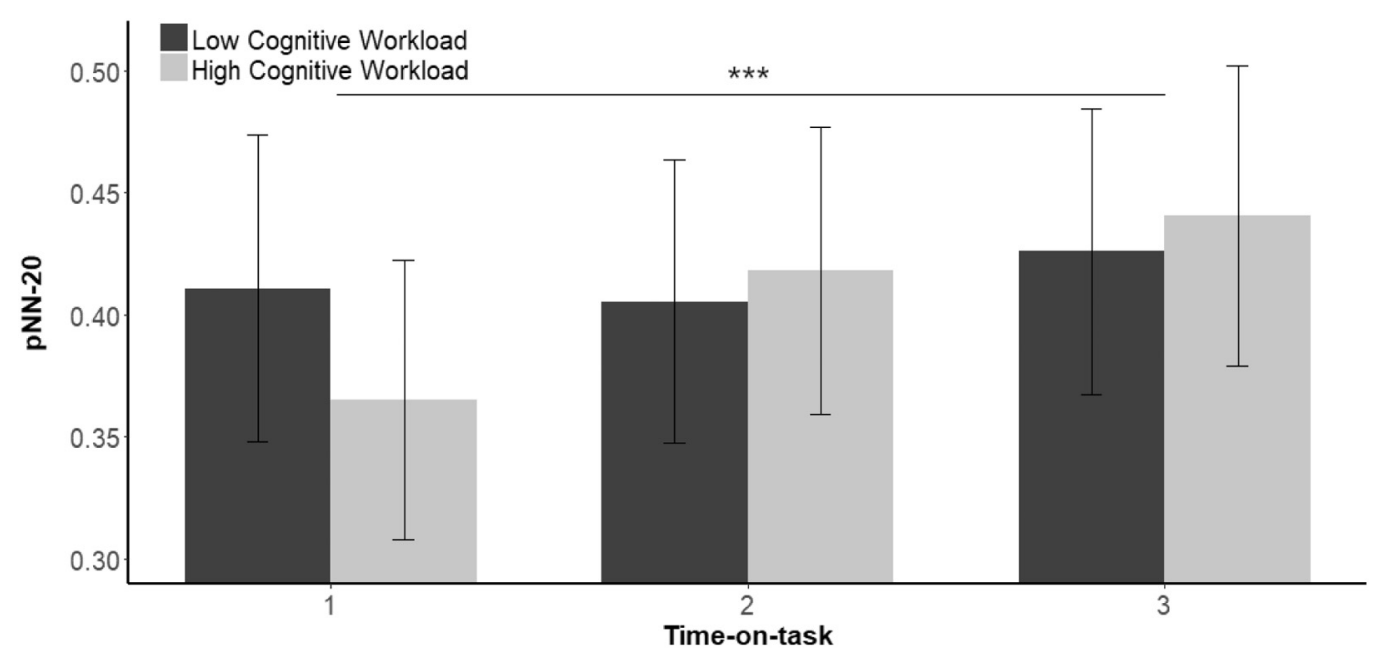

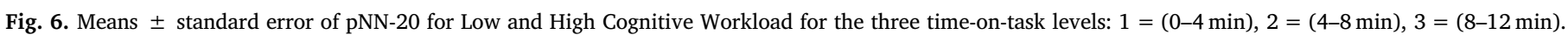
$* * * p<.001$.

(HCW), implying a globally stronger sympathetic activity, closely linked to the rise of catecholamines secretion (Heine et al., 2017).

The interaction between cognition and emotion described in the work of Patel et al. (2016), where the authors showed that induced arousal affected differently working memory depending on the task difficulty and, reciprocally, the increase of the cognitive demand lightened the physiological responses linked to anxiety, was not evidenced in the studied HRV parameters. Nonetheless, the intrinsic stress generated by the task difficulty, which is considered as an influent factor for mental workload (Hsu et al., 2015), might have slightly biased the results. In any case, as previously demonstrated by several other studies (Causse et al., 2011; Wei et al., 2014; Mandrick et al., 2016), our results confirmed the sensitivity of HR to cognitive demand, with increased HR when the secondary task was more complex.

\subsection{Time-on-task effect on ECG}

Concurrently to the emotion and cognition factor analysis, it was important to take into account the cardiovascular activity evolution over time during the flight simulation, since its variations could increase the difficulty of accurately computing baselines of the ECG features. Note that time-on-task refers here to both emotional habituation and training to the tasks, as the experiment did not allow to separate the respective influence of these two factors. In addition, apart from the inherent non-stationarity ECG, short-term variations of the instantaneous parameter values may have appeared due to different flight scenario instructions (e.g. changing the direction, reducing speed, etc.), which required an increase of the vigilance level, possibly altering the cardiac response in different time periods (Luque-Casado et al., 2016; Tremayne and Barry, 2001). A prominent peak in HR was found immediately when the difficulty of the secondary task increased (beginning of HCW). By contrast, increases of pNN- $x$ were observed along the HCW conditions.

According to Luque-Casado et al. (2016), a decrease of RMSSD over time would be expected due to mental fatigue. However, an increase of HRV was found, arguably due to the training effect and the consequent decrease of cognitive effort (Mandrick et al., 2016). With regards to pNN- $x$, when the time-on-task factor was analysed, pNN-20 showed a monotonic increase during the HCW conversely to the LCW condition, where the minimum was reached at the middle of time course (Fig. 6). On the other hand, consistently with the conclusions reported in Mietus et al. (2002) for pathological populations, pNN-20 showed more notable differences than pNN-50 to determine inter-condition differences. Given that our population was constituted by healthy participants and that the crucial comparisons were intra-subject, no more fixed values apart from the typical 20 and $50 \mathrm{~ms}$ were tested to avoid over-fitted results. Nevertheless, in order to get an equitable, but intra-subject customized measure, pNN-SD was also analysed, where SD was adaptive to the individual differences. However, no better results were found, returning to the use of fixed time intervals as a better option. Therefore, taking a $20 \mathrm{~ms}$ as threshold appears as a good trade-off for cognitive workload analysis by means of pNN.

\section{Limitations of the study}

One of the main limitations of this work is that respiratory rate variation could be one of the confounding factors intervening in the time-on-task effect. Therefore, we have to be cautious to claim the link between a pure cardiac activity and cognitive workload. In any case, to evaluate the HR measure usefulness to implement monitoring systems, the origin of its modulation is not essential. It would be possible to build a robust classifier even if the HR changes were due to respiratory changes, as long as they covariate over time. Either way, in future works, mainly focusing on determining the heart physiology, it would be desirable to apply a methodology including respiratory estimation (Thayer et al., 1996).

While cognitive workload was counterbalanced within the two dual task scenarios, the social stressor was always administered during the second dual-task scenario. Given the potential long term effect of increased emotional arousal, we wanted to avoid an emotional contamination on the second scenario. Yet, the lack of counterbalancing limits the possibility to distinguish an effect of emotion from an effect of training/fatigue, which is a limitation of this study.

The technical problems which prevented the recording of behavioural data from every subject could have biased the results concerning the RT. Either way, the significant differences between conditions with different levels of cognitive workload enable to assume that the difficulty of the tasks was different. Nonetheless, a subjective measure of the cognitive workload, such as the NASA-TLX test would have been suitable to confirm this assumption.

\section{Conclusions}

During the flight simulator sessions, we manipulated emotion with a social stressor and cognitive workload with the difficulty of a secondary task, carried out in parallel to flight simulation. We assessed the impact of these two factors on behavioural performance (obtained in the piloting task as well as in the secondary task), and cardiovascular activity. 
Behavioural results of the secondary task confirmed that both emotional and cognitive factors significantly impacted behaviour. We found that a moderate social stress can enhance motivation and performance with a decrease of omission errors. On the contrary, a high mental workload condition was deleterious to performance with increased reaction times.

From a physiological standpoint, our results confirmed the sensitivity of the HR to cognitive demand and time-on-task (mainly due to a training effect), with increased HR when the task was more difficult and decreased HR with training. Training was also associated with an increased HRV, with increased values along the flight scenario time course. The inclusion of other physiological signals such as electrodermal activity, which could be feasible to incorporate into a cockpit, is desirable in order to extract additional robust features representing the general cognitive and emotional state of pilots.

\section{Acknowledgements}

This work was supported by the French Research National Agency and the French Defence Procurement Agency via the Accompagnement Spécifique des travaux de Recherches et d'innovation Défense (ASTRID), project ANR-13-ASTR-0036.

\section{References}

Allsop, J., Gray, R., 2014. Flying under pressure: effects of anxiety on attention and gaze behavior in aviation. J. Appl. Res. Mem. Cogn. 3 (2), 63-71.

Besson, P., Maiano, C., Bringoux, L., Marqueste, T., Mestre, D.R., Bourdin, C., Dousset, E., Durand, M., Vercher, J., 2012, September. Cognitive workload and affective state: a computational study using Bayesian networks. In: Intelligent Systems (IS), 2012 6th IEEE International Conference. IEEE, pp. 140-145.

Blanchard, R.J., Hebert, M., Sakai, R.R., McKittrick, C., Henrie, A., Yudko, E., McEwen, B.S., Blanchard, D.C., 1998. Chronic social stress: changes in behavioral and physiological indices of emotion. Aggress. Behav. 24 (4), 307-321.

Blogut, A., 2015. Stressing Factors in Aviation. Scientific Research \& Education in the Air Force - AFASES.

Causse, M., Sénard, J.M., Démonet, J.F., Pastor, J., 2010. Monitoring cognitive and emotional processes through pupil and cardiac response during dynamic versus logical task. Appl. Psychophysiol. Biofeedback 35 (2), 115-123.

Causse, M., Baracat, B., Pastor, J., Dehais, F., 2011. Reward and uncertainty favor risky decision-making in pilots: evidence from cardiovascular and oculometric measurements. Appl. Psychophysiol. Biofeedback 36 (4), 231-242.

Causse, M., Dehais, F., Péran, P., Sabatini, U., Pastor, J., 2013. The effects of emotion on pilot decision-making: a neuroergonomic approach to aviation safety. Trans. Res. C Emerg. Technol. 33, 272-281.

Causse, M., Chua, Z., Peysakhovich, V., Campo, N., Matton, N., 2017. Mental workload and neural efficiency quantified in the prefrontal cortex using fNIRS. Sci. Rep. 7 (1), 5222.

Critchley, H.D., 2005. Neural mechanisms of autonomic, affective, and cognitive integration. J. Comp. Neurol. 493 (1), 154-166.

Critchley, H.D., Eccles, J., Garfinkel, S.N., 2013. Interaction between cognition, emotion, and the autonomic nervous system. Handb. Clin. Neurol. 117, 59-77.

Durantin, G., Gagnon, J.F., Tremblay, S., Dehais, F., 2014. Using near infrared spectroscopy and heart rate variability to detect mental overload. Behav. Brain Res. 259, $16-23$.

Feda, D.M., Roemmich, J.N., 2016. Effect of interpersonal and cognitive stressors on habituation and the utility of heart rate variability to measure habituation. Stress. Health 32 (4), 320-327.

Gaetan, S., Dousset, E., Marqueste, T., Bringoux, L., Bourdin, C., Vercher, J.L., Besson, P., 2015. Cognitive workload and psychophysiological parameters during multitask activity in helicopter pilots. Aerosp. Med. Hum. Perform. 86 (12), 1052-1057.

Gaillard, A., Wientjes, C., 1994. Mental load and work stress as two types of energy mobilization. Work Stress. 8 (2), 141-152.

Gray, R., Gaska, J., Winterbottom, M., 2016. Relationship between sustained, orientated, divided, and selective attention and simulated aviation performance: training \& pressure effects. J. Appl. Res. Mem. Cogn. 5 (1), 34-42.

Heine, T., Lenis, G., Reichensperger, P., Beran, T., Doessel, O., Deml, B., 2017. Electrocardiographic features for the measurement of drivers' mental workload. Appl. Ergon. 61, 31-43.

Hsu, B.W., Wang, M.J.J., Chen, C.Y., Chen, F., 2015. Effective indices for monitoring mental workload while performing multiple tasks. Percept. Mot. Skills 121 (1), 94-117.

Jorna, P.G.A.M., 1993. Heart rate and workload variations in actual and simulated flight. Ergonomics 36 (9), 1043-1054.

Lazarus, R.S., 2006. Stress and Emotion: A New Synthesis. Springer Publishing Company.

Luque-Casado, A., Perales, J.C., Cárdenas, D., Sanabria, D., 2016. Heart rate variability and cognitive processing: the autonomic response to task demands. Biol. Psychol. 113, 83-90.

Mandrick, K., Peysakhovich, V., Rémy, F., Lepron, E., Causse, M., 2016. Neural and psychophysiological correlates of human performance under stress and high mental workload. Biol. Psychol. 121, 62-73.

Mansikka, H., Simola, P., Virtanen, K., Harris, D., Oksama, L., 2016. Fighter pilots' heart rate, heart rate variation and performance during instrument approaches. Ergonomics 59 (10), 1344-1352.

Martinent, G., Ferrand, C., Guillet, E., Gautheur, S., 2010. Validation of the French version of the Competitive State Anxiety Inventory-2 Revised (CSAI-2R) including frequency and direction scales. Psychol. Sport Exerc. 11 (1), 51-57.

Massaro, S., Pecchia, L., 2016. Heart rate variability (HRV) analysis a methodology for organizational neuroscience. Organ. Res. Methods. http://dx.doi.org/10.1177/ 1094428116681072 .

Mehler, B., Reimer, B., Coughlin, J.F., 2012. Sensitivity of physiological measures for detecting systematic variations in cognitive demand from a working memory task: an on-road study across three age groups. Hum. Factors 54 (3), 396-412.

Mietus, J.E., Peng, C.K., Henry, I., Goldsmith, R.L., Goldberger, A.L., 2002. The pNNx files: re-examining a widely used heart rate variability measure. Heart 88 (4), 378-380.

Muth, E.R., Moss, J.D., Rosopa, P.J., Salley, J.N., Walker, A.D., 2012. Respiratory sinus arrhythmia as a measure of cognitive workload. Int. J. Psychophysiol. 83 (1), 96-101.

Patel, N., Vytal, K., Pavletic, N., Stoodley, C., Pine, D.S., Grillon, C., Ernst, M., 2016. Interaction of threat and verbal working memory in adolescents. Psychophysiology 53 (4), 518-526.

Puma, S., Matton, N., Paubel, P.V., Raufaste, É., El-Yagoubi, R., 2018. Using theta and alpha band power to assess cognitive workload in multitasking environments. Int. J. Psychophysiol. 123, 111-120.

Salas, E., Maurino, D., Curtis, M. (Eds.), 2010. Human Factors in Aviation, 2nd edition. Academic Press.

Scherer, K.R., 2005. What are emotions? And how can they be measured? Soc. Sci. Inf. 44 (4), 695-729.

Sevenster, D., Hamm, A., Beckers, T., Kindt, M., 2015. Heart rate pattern and resting heart rate variability mediate individual differences in contextual anxiety and conditioned responses. Int. J. Psychophysiol. 98 (3), 567-576.

Socha, V., Schlenker, J., Kal'avksý, P., Kutilek, P., Socha, L., Szabo, S., Smrčka, P., 2015, January. Effect of the change of flight, navigation and motor data visualization on psychophysiological state of pilots. In: 2015 IEEE 13th International Symposium on Applied Machine Intelligence and Informatics (SAMI). IEEE, pp. 339-344.

Spielberger, C.D., Gorsuch, R.L., Lushene, R., Vagg, P.R., Jacobs, G., 1983. Manual for the State-trait Anxiety Inventory (Form Y): Self-evaluation Questionnaire. Consulting Psychologists Press, Palo Alto, CA.

Staal, M.A., 2004. Stress, Cognition, and Human Performance: A Literature Review and Conceptual Framework.

Stikic, M., Berka, C., Levendowski, D.J., Rubio, R.F., Tan, V., Korszen, S., Barba, D., Wurzer, D., 2014. Modeling temporal sequences of cognitive state changes based on a combination of EEG-engagement, EEG-workload, and heart rate metrics. Front. Neurosci. 8, 242-255.

Stuiver, A., Brookhuis, K.A., de Waard, D., Mulder, B., 2014. Short-term cardiovascular measures for driver support: increasing sensitivity for detecting changes in mental workload. Int. J. Psychophysiol. 92 (1), 35-41.

Thayer, J.F., Peasley, C., Muth, E.R., 1996. Estimation of respiratory frequency from autoregressive spectral analysis of heart period. Biomed. Sci. Instrum. 32, 93-99.

Tichon, J.G., Wallis, G., Riek, S., Mavin, T., 2014. Physiological measurement of anxiety to evaluate performance in simulation training. Cogn. Tech. Work 16 (2), 203-210.

Tremayne, P., Barry, R.J., 2001. Elite pistol shooters: physiological patterning of best vs. worst shots. Int. J. Psychophysiol. 41 (1), 19-29.

Vecchiato, G., Borghini, G., Aricò, P., Graziani, I., Maglione, A.G., Cherubino, P., Babiloni, F., 2016. Investigation of the effect of EEG-BCI on the simultaneous execution of flight simulation and attentional tasks. Med. Biol. Eng. Comput. 54 (10), 1503-1513.

Wang, J., Rao, H., Wetmore, G.S., Furlan, P.M., Korczykowski, M., Dinges, D.F., Detre, J.A., 2005. Perfusion functional MRI reveals cerebral blood flow pattern under psychological stress. Proc. Natl. Acad. Sci. U. S. A. 102 (49), 17804-17809.

Wei, Z., Zhuang, D., Wanyan, X., Liu, C., Zhuang, H., 2014. A model for discrimination and prediction of mental workload of aircraft cockpit display interface. Chin. J. Aeronaut. 27 (5), 1070-1077.

Wickens, C.D., 2008. Multiple resources and mental workload. Hum. Factors J. Hum. Factors Ergon. Soc. 50 (3), 449-455.

Young, M.S., Brookhuis, K.A., Wickens, C.D., Hancock, P.A., 2015. State of science: mental workload in ergonomics. Ergonomics 58 (1), 1-17. 